\title{
Novel Sort-Free Detector with Modified Real-Valued Decomposition (M-RVD) Ordering in MIMO Systems
}

\author{
Kiarash Amiri, Chris Dick, Raghu Rao, Joseph R. Cavallaro \\ kiaa@rice.edu, chris.dick@xilinx.com, rrao@xilinx.com, cavallar@ rice.edu
}

\begin{abstract}
K-best MIMO detection technique is the prominent method of simplifying the detection complexity in MIMO systems while maintaining BER performance comparable with the optimum maximum-likelihood (ML) detection technique. However, sorting the candidate nodes in the tree search of the conventional K-best detection can take a significant number of cycles which would reduce the achievable data rate of the detector. In order to reduce this delay, and keep high performance at the same time, we propose using a novel sort-free based MIMO detector which avoids the demanding sorting step. Moreover, this detector utilizes a novel modified real-valued decomposition (M-RVD) ordering that, when compared to the conventional real valued decomposition scheme, can improve the BER performance at no extra computational cost. We show that our proposed detector can outperform the conventional K-best detector with a smaller combination of computation and latency requirements.
\end{abstract}

\section{INTRODUCTION}

Multiple-input multiple-output (MIMO) systems have been one of the main research topics in wireless communication for their capability of achieving very high spectral efficiencies [1] as well as offering more robustness to mitigate the inherent fading in wireless channels. However, any effort to design a MIMO-based transceiver needs to address the detection problem. The complexity of the optimum detector, i.e. maximum-likelihood (ML) receiver, grows exponentially as more antennas are used at the transmitter, and as higher order modulation schemes are adopted. In order to reduce the complexity, sphere detection [2], and its K-best variation, has been proposed [3], analyzed [4] and implemented [5], [6], [7], [8], [9].

As MIMO solutions become more popular and are incorporated into different wireless standards, such as IEEE 802.11n, IEEE 802.16e and upcoming 3GPP LTE, it is crucial to investigate methods to further reduce the complexity of detection while maintaining high BER performance. Conventional Kbest MIMO detectors typically require long delay cycles for sorting steps. For instance, for a multi-stage real-valued based K-best detector for a 16-QAM MIMO system, a bubble sorter needs more than 40 cycles if the detector parameter, $K$, is set to 10 . This long list size introduces a large delay for the processing of the next stage. Moreover, in order to achieve higher reliability, it is important to come up with a cost-free ordering scheme that would lead to a further error performance improvement of the system.

In this paper, we propose a novel K-best type MIMO detector, with two major contributions, namely by:

1. adopting a sort-free strategy in interfacing the detection stages which avoids the need for exhaustive long sortings,

2. utilizing a cost-free novel real-valued decomposition (MRVD) ordering scheme which improves the performance.

The paper is organized as follows: Section II introduces the system model and the conventional MIMO detector, section III introduces the proposed MIMO detector. The complexity issues and comparisons are discussed in section IV, and the simulation results are presented in section V. Finally, the papers is concluded with section VI.

\section{SYSTEM MODEL}

We consider a MIMO system with $M_{T}$ transmit and $M_{R}$ receive antennas. The input-output model is captured by

$$
\tilde{\mathbf{y}}=\tilde{\mathbf{H}} \tilde{\mathbf{s}}+\tilde{\mathbf{n}}
$$

where $\tilde{\mathbf{H}}$ is the complex-valued $M_{R} \times M_{T}$ channel matrix, $\tilde{\mathbf{s}}=\left[\tilde{s}_{1}, \tilde{s}_{2}, \ldots, \tilde{s}_{M_{T}}\right]^{T}$ is the $M_{T}$-dimensional transmitted vector whose elements are chosen from a complex-valued constellation $\Omega$ of the order $w=|\Omega|, \tilde{\mathbf{n}}$ is the circularly symmetric complex additive white Gaussian noise vector of size $M_{R}$ and $\tilde{\mathbf{y}}=\left[\tilde{y}_{1}, \tilde{y}_{2}, \ldots, \tilde{y}_{M_{R}}\right]^{T}$ is the $M_{R}$-element received vector. The preceding MIMO equation can be decomposed into real-valued numbers as follows [10]:

$$
\mathbf{y}=\mathbf{H} \mathbf{s}+\mathbf{n}
$$

corresponding to

$$
\left(\begin{array}{c}
\Re(\tilde{\mathbf{y}}) \\
\Im(\tilde{\mathbf{y}})
\end{array}\right)=\left(\begin{array}{cc}
\Re(\tilde{\mathbf{H}}) & -\Im(\tilde{\mathbf{H}}) \\
\Im(\tilde{\mathbf{H}}) & \Re(\tilde{\mathbf{H}})
\end{array}\right)\left(\begin{array}{c}
\Re(\tilde{\mathbf{s}}) \\
\Im(\tilde{\mathbf{s}})
\end{array}\right)+\left(\begin{array}{c}
\Re(\tilde{\mathbf{n}}) \\
\Im(\tilde{\mathbf{n}})
\end{array}\right)
$$

with $M=2 \cdot M_{T}$ and $N=2 \cdot M_{R}$ presenting the dimensions of the new system.

We call the ordering in (2), the conventional ordering. Using the conventional ordering, all the computations can be performed using only real values. Note that after real-valued decomposition, each $s_{i}$ in $\mathbf{s}$ is chosen from a set of real numbers, $\Omega^{\prime}$, with $w^{\prime}=\sqrt{w}$ elements. 
The maximum-likelihood (ML) detector, which is optimum for such a system, minimizes $\|\mathbf{y}-\mathbf{H s}\|^{2}$ over all the possible combinations of the $\mathrm{s}$ vector. However, the ML detection requires exhaustive exponentially growing search among all the candidates, and is not practically feasible in a large dimension MIMO receiver. In order to avoid the significant overhead of the ML detection, the distance norm can be simplified [11] as follows:

$$
\begin{aligned}
D(\tilde{\mathbf{s}}) & =\|\mathbf{y}-\mathbf{H} \mathbf{s}\|^{2} \\
& =\left\|\mathbf{Q}^{\mathbf{H}} \mathbf{y}-\mathbf{R s}\right\|^{2}=\sum_{i=M}^{1}\left|y_{i}{ }^{\prime}-\sum_{j=i}^{M} R_{i, j} s_{j}\right|^{2}
\end{aligned}
$$

where $\mathbf{H}=\mathbf{Q R}$ represents the channel matrix $\mathrm{QR}$ decomposition, $\mathbf{R}$ is an upper triangular matrix, $\mathbf{Q Q}^{\mathbf{H}}=\mathbf{I}$ and $\mathbf{y}^{\prime}=\mathbf{Q}^{\mathbf{H}} \mathbf{y}$.

The norm in (4) can be computed in $M$ iterations starting with $i=M$. When $i=M$, i.e. the first iteration, the initial partial norm is set to zero, $T_{M+1}\left(\mathbf{s}^{(M+1)}\right)=0$. Using the notation of [5], at each iteration the Partial Euclidean Distances (PEDs) at the next levels are given by

$$
T_{i}\left(\mathbf{s}^{(i)}\right)=T_{i+1}\left(\mathbf{s}^{(i+1)}\right)+\left|e_{i}\left(\mathbf{s}^{(i)}\right)\right|^{2}
$$

with $\mathbf{s}^{(i)}=\left[s_{i}, s_{i+1}, \ldots, s_{M}\right]^{T}$, and $i=M, M-1, \ldots, 1$, where

$$
\left|e_{i}\left(\mathbf{s}^{(i)}\right)\right|^{2}=\left|y_{i}{ }^{\prime}-R_{i, i} s_{i}-\sum_{j=i+1}^{M} R_{i, j} s_{j}\right|^{2}
$$

One can envision this iterative algorithm as a tree traversal with each level of the tree corresponding to one $i$ value, and each node having $w^{\prime}$ children.

The tree traversal can be performed in a breadth-first manner. At each level, only the best $K$ nodes, i.e. the $K$ nodes with the smallest $T_{i}$, are chosen for expansion. This type of detector is generally known as the $K$-best detector. Note that such a detector requires sorting a list of size $K \times w^{\prime}$ to find the best $K$ candidates. For instance, for a 16-QAM system with $K=10$, this requires sorting a list of size $K \times w^{\prime}=10 \times 4=40$ at most of the tree levels. This introduces a long delay for the next processing block in the detector unless a highly parallel sorter is used. Highly parallel sorters, on the other hand, consist of a large number of compare-select blocks, and result in dramatic area increase.

\section{Novel Sort-Free Detector}

In this section, we propose a novel MIMO detector, which utilizes a sort-free strategy and is based on a new modified real-valued decomposition (M-RVD) scheme. The proposed technique significantly reduces the delay of the detector, that would have otherwise existed due to the large sorting delay.

\section{A. Tree Traversal for Sort-Free Detection}

We propose a sort-free detector to mitigate the sorting overhead. Therefore, the long sorting operation is reduced to a minimum-finding function. The following table describes the details of this algorithm:

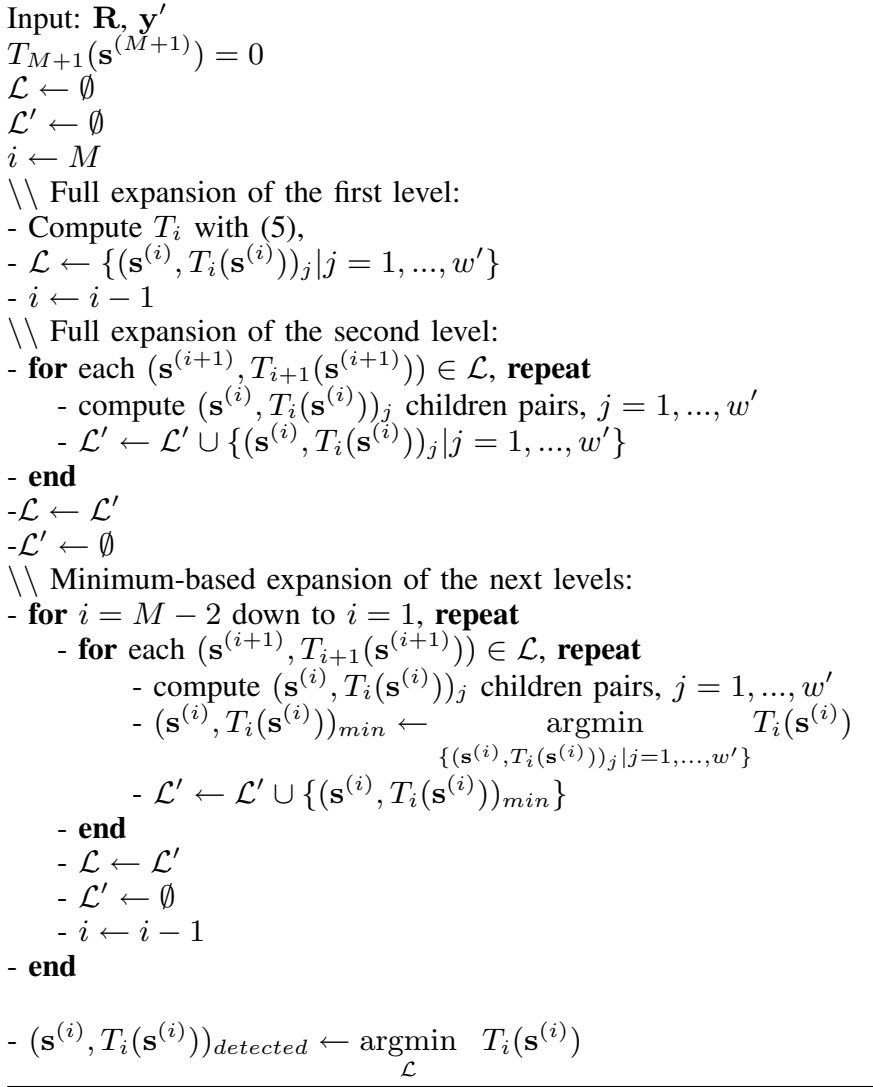

An example of this algorithm is illustrated in Figure 1 for a $3 \times 3,16$-QAM system. Note that as described above, the first two levels are fully expanded to guarantee high performance; whereas for the following levels, only the best candidate in the children list of a parent node is expanded. In other words, after passing the first two levels, $w$ nodes are expanded, and for each of those $w$ nodes, the best children node among its $w^{\prime}$ children nodes are selected as the survived node. Therefore, the new node list would contain $w$ nodes in the third level. These $w$ nodes are expanded in a similar way to the fourth level, and this procedure continues until the very last level, where the minimum-distance node is taken as the detected node. Thus, the long sorting of $K$-best is replaced by significantly less complicated minimum-finding over smaller sets.

\section{B. Modified Real-Valued Decomposition (M-RVD) Ordering}

We propose using a novel real-valued decomposition (MRVD) ordering which improves the BER performance compared to the ordering given in Eq. (2). The new decomposition is summarized as:

$$
\hat{\mathbf{y}}=\hat{\mathbf{H}} \hat{\mathbf{s}}+\hat{\mathbf{n}}
$$




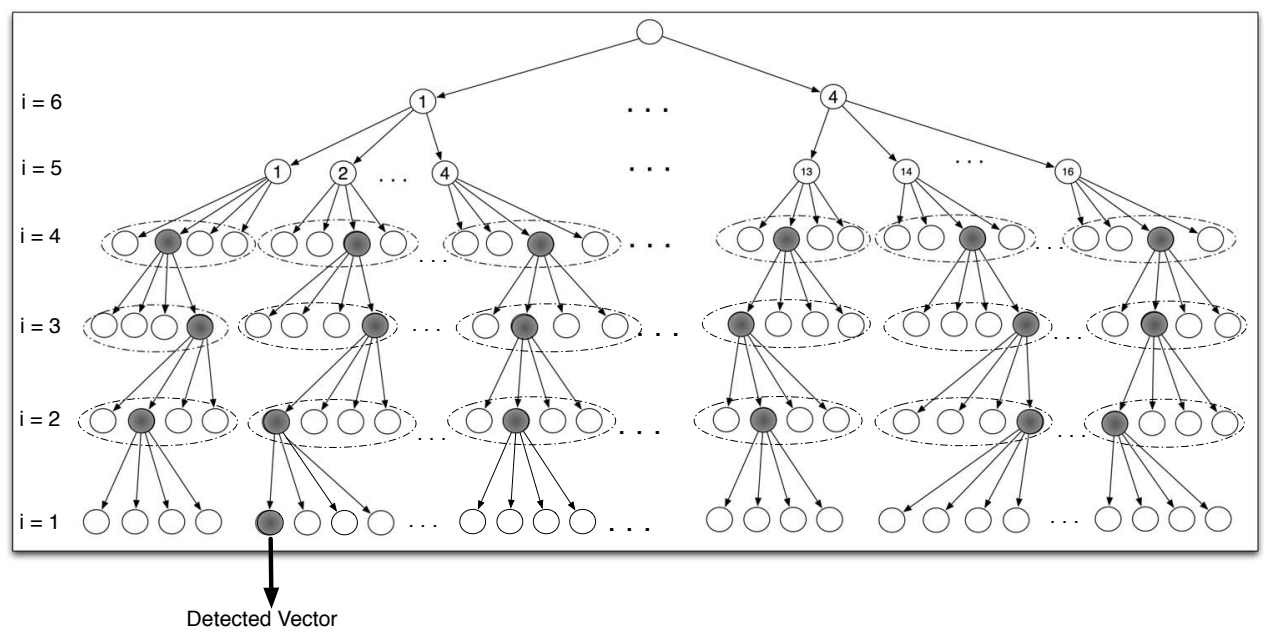

Fig. 1. Sort-free algorithm for an example 16-QAM, $3 \times 3$ system. The topmost two levels are fully expanded. The nodes marked with black are the minimum in their own set, where each set is denoted by dashed line. Note that because of the real-valued decomposition, each node has only $\sqrt{16}=4$ children. Also, the number of tree levels are $M=2 \times M_{T}=6$.

or,

$$
\left(\begin{array}{c}
\Re\left(\tilde{y}_{1}\right) \\
\Im\left(\tilde{y}_{1}\right) \\
\Re\left(\tilde{y}_{2}\right) \\
\Im\left(\tilde{y}_{2}\right) \\
\cdot \\
\cdot \\
\cdot \\
\Re\left(\tilde{y}_{M_{R}}\right) \\
\Im\left(\tilde{y}_{M_{R}}\right)
\end{array}\right)=\hat{\mathbf{H}}\left(\begin{array}{c}
\Re\left(\tilde{s}_{1}\right) \\
\Im\left(\tilde{s}_{1}\right) \\
\Re\left(\tilde{s}_{2}\right) \\
\Im\left(\tilde{s}_{2}\right) \\
\cdot \\
\cdot \\
\cdot \\
\Re\left(\tilde{s}_{M_{T}}\right) \\
\Im\left(\tilde{s}_{M_{T}}\right)
\end{array}\right)+\left(\begin{array}{c}
\Re\left(\tilde{n}_{1}\right) \\
\Im\left(\tilde{n}_{1}\right) \\
\Re\left(\tilde{n}_{2}\right) \\
\Im\left(\tilde{n}_{2}\right) \\
\cdot \\
\cdot \\
\cdot \\
\Re\left(\tilde{n}_{M_{R}}\right) \\
\Im\left(\tilde{n}_{M_{R}}\right)
\end{array}\right)
$$

where $\hat{\mathbf{H}}$ is the permuted channel matrix of Eq. (3) whose columns are reordered to match the other vectors of the new decomposition ordering in Eq. (7). It is worth noting that there is no extra computational cost associated with this novel ordering.

Note that with the modified real-valued decomposition (MRVD) ordering, the first two levels correspond to the in-phase and quadrature parts of the same complex symbol; whereas in the conventional real-valued decomposition scenario, the first two levels of the tree correspond to the quadrature parts of two different complex symbols. A careful look at the tree traversal scheme of the preceding section shows that since the first two levels of the tree are fully expanded, the error performance of the scheme heavily depends on the third level of the tree. Therefore, rather than using the magnitude of $R_{M, M}$ as a metric to choose the decomposition ordering scheme, which justifies the conventional real-valued decomposition (RVD) [12], we need to look at the behavior of the third lowest diagonal element of the $\mathbf{R}$ matrix. As demonstrated in Figure 2, there is an increase in the magnitude of $R_{M-2, M-2}$ when using M-RVD, hence M-RVD is a better choice than the conventional RVD. The impact of M-RVD on the BER performance is discussed in the next sections.

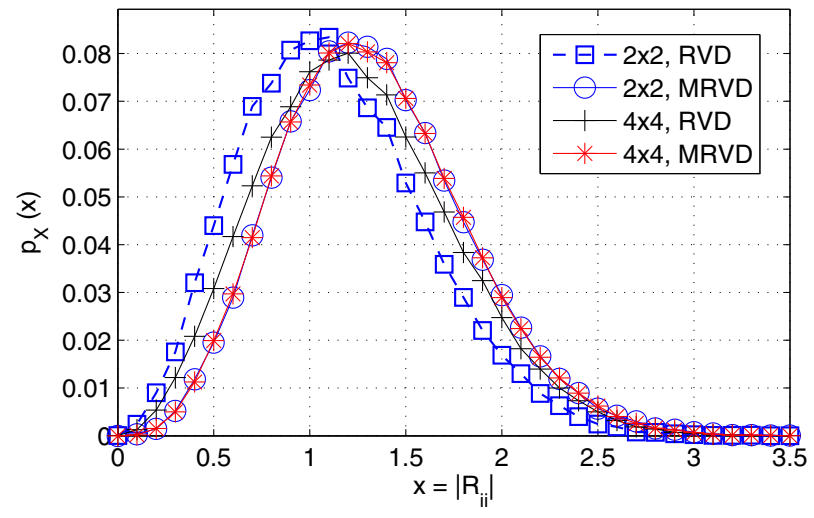

Fig. 2. Probability density function of the $R_{6,6}$ for $4 \times 4$ and $R_{2,2}$ for $2 \times 2$ when either conventional RVD or the proposed RVD are used. Note the shift of the curves when M-RVD is used.

\section{COMPLEXITY COMPARISON}

In order to compare the complexity of the proposed MIMO detector, described in the preceding section, versus the conventional K-best technique, we consider the number of operations, the relative latency reduction, and the architecture advantages of the proposed detector.

\section{A. Number of Operations}

In this section, we compute the number of operations required to complete the detection process. Since the channel matrix typically changes at a much slower rate than the received signal vector, we make the assumption that simple channel matrix operations, e.g. $R_{i j} s_{j}$ computations, are performed in a separate pre-processing unit. Note that this simply involves shift-add operations with $s_{j} \in \Omega^{\prime}$. Also, as suggested in [5], we make the assumption that all the PED 
TABLE I

COMPARISON OF THE OPERATION COUNTS BETWEEN THE CONVENTIONAL $K$-BEST AND THE PROPOSED SORT-FREE DETECTOR.

\begin{tabular}{|c|c|c|}
\hline & $K$-best & Proposed sort-free detector \\
\hline \hline Addition & $2 w^{\prime}+2 w+2 K w^{\prime}(M-2)+K(M(M-1) / 2-1)$ & $2 w^{\prime}+w+w \cdot w^{\prime}(M(M+1) / 2-3)$ \\
\hline Compare-select & $f_{K}\left(K w^{\prime}\right)(M-3)+f_{K}(w)+f_{1}\left(K w^{\prime}\right)$ & $w^{\prime} f_{1}\left(w^{\prime}\right)(M-3)+f_{1}(w)$ \\
\hline Multiplication & $w^{\prime}+w+K w^{\prime}(M-2)$ & - \\
\hline
\end{tabular}

norms are approximated by $\ell^{1}$-norms to avoid the squarers and multipliers. Therefore, the only major high rate detector operations, are compare-select for either sorting or minimumfindings, addition and multiplication.

Given the above assumptions, the total number of operations for the $K$-best scenario and the proposed sort-free scheme are given in Table I. Note that in order to achieve minimum latency, we make the assumption that both detectors use cascaded minimum-finders to sort a list. Therefore, in order to find the best $K$ elements of a list of size $l ; K$ cascaded minimum finders are required. So, the number of operations required to sort the best $K$ candidates of a list of size $l$, denoted by $f_{K}(l)$ in Table $\mathrm{I}$, is given by

$$
f_{K}(l)=K \times l-\frac{K(K+1)}{2} .
$$

In order to compute the final operation count, comparators are assumed to have unit complexity, and adders to have twice complexity as that of comparators. Multipliers, to implement the squarers, are furthermore, assumed to be ten times more complex than additions. It is worth noting that other relative complexity coefficients would yield similar general results. Based on these relative complexities, the number of operations are plotted for different numbers of antennas in Figure 3. Note that except for small $K$ values, the computation overhead of the conventional $K$-best scheme is considerably more than the proposed sort-free scheme. More details on the BER performance comparisons will be presented in section V.

\section{B. Latency}

High latency decreases the data rate in feedback based receivers. For instance, for iterative detector/decoder structures, where the detector uses the feedback data from the decoder to improve the detection performance, higher detection/decoding latency reduces the data rate significantly. A similar argument applies to the overall receiver throughput when the interaction between the physical layer and MAC layer takes more cycles due to the higher physical layer latency. We compare the latency overhead of our proposed detector versus the conventional $K$-best detector, and show that the sort-free technique introduces significant latency reduction.

Note that if the detectors are fully parallelized for enhancing data rates, the conventional $K$-best detector requires $K$ successive minimum finders; whereas, the proposed sort-free detector requires only one level of minimum finder as it only needs to find the minimum, i.e. sorting with $K=1$. Thus, if we assume full parallelism for both types of detectors, the latency of the sorter that connects one of the middle levels of the tree to the next level is given in Table II.

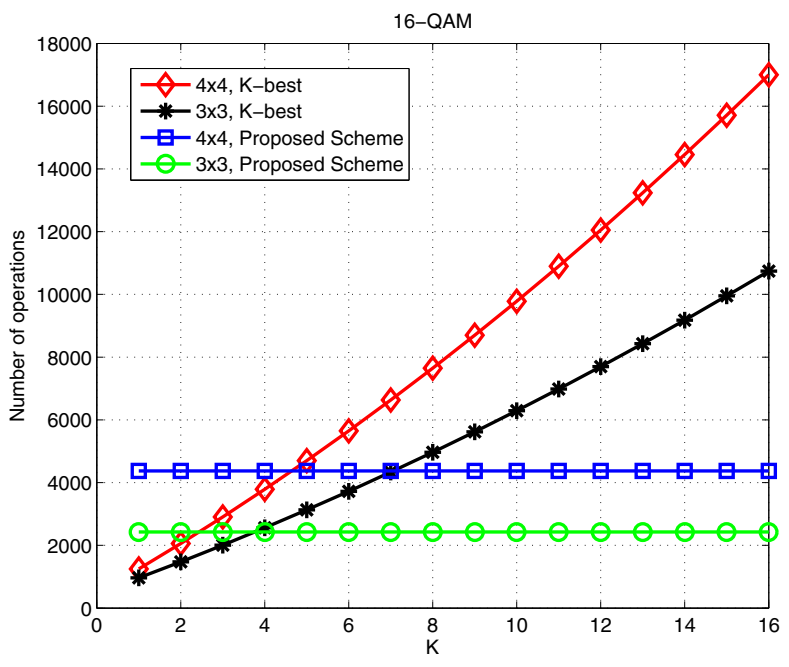

Fig. 3. Comparison of the number of operations between the proposed scheme and $K$-best for different values of $K$ and different number of antennas. The 16-QAM modulation is assumed.

Notice the significant latency reduction that the proposed sort-free detector promises for the sorting after each level. Also, note that Table II represents only the latency of one level; thus, for a $4 \times 4$ system, there would be $M-3=2 M_{T}-3=5$ of such sorters, see Table I.

\section{Architecture}

The common K-best sorting requires a bubble-sort architecture [9]. In this architecture, all the nodes need to be passed into the sorter sequentially, and the process of the next level of the tree can not start until all the $K \times w^{\prime}$ nodes are passed through the sequential sorter. Even semi-parallel sorters, still require large area and cycles, to finish the detection process, see Tables I and II and Figure 3. With the sort-free technique, all the long size sortings are avoided. Moreover, the sort-free technique is amenable to parallelizing with less overhead than the $K$-best technique.

\section{Simulation Results}

For the BER simulations, the Rayleigh fading channel model is assumed, and the channel matrix is independent for each new transmission. The BER results of $4 \times 4$ and $3 \times 3$ systems are compared for a 16-QAM modulation scheme. Note that in order to conduct a fair performance comparison, the $K$ values are chosen such that the $K$-best technique has similar number of operations as that of the proposed sort-free scheme, 
TABLE II

COMPARISON OF THE SORTING LATENCY, PER LEVEL, BETWEEN THE CONVENTIONAL $K$-BEST AND THE PROPOSED SORT-FREE DETECTOR. ALL THE lOg FUNCTIONS ARE IN BASE 2 , I.E. $\log _{2}$.

\begin{tabular}{|c|c|c|}
\hline & $K$-best & Proposed sort-free detector \\
\hline Latency & $\sum_{m=0}^{K-1}\left\lceil\log \left(K w^{\prime}-m\right)\right\rceil$ & $\left\lceil\log w^{\prime}\right\rceil$ \\
\hline$\overline{\text { Example (16-QAM, } K=4)}$ & 16 & $\overline{2}$ \\
\hline Example (16-QAM, $K=5$ ) & 24 & 2 \\
\hline
\end{tabular}

see Figure 3. Therefore, based on the results shown in Figure 3 and Table $\mathrm{I}, K$ is set to 5 and 4 for the $4 \times 4$ and $3 \times 3$ systems, respectively.

The BER simulation results of Figure 4 suggest that the proposed sort-free scheme can improve the BER performance more than $5 \mathrm{~dB}$ compared to the conventional $K$-best technique in higher SNR regimes. Note that it was shown in the preceding sections that for a $4 \times 4$ case, the $K=5$ scheme requires similar computational complexity as that of the sortfree scheme, and it requires 12 times more latency for sorting in each level compared to the proposed sort-free scheme. A similar argument holds for a $3 \times 3$ system when $K=4$. It is also worth noting that in both cases, the M-RVD ordering plays an important role in improving the performance.

\section{CONCLUSION}

In this paper, we proposed a novel MIMO detector which improves the performance and reduces the complexity when compared to the conventional $K$-best MIMO detector. We showed that avoiding the sorting step and adopting a novel decomposition ordering, our scheme demonstrates better BER behavior with similar complexity.

\section{ACKNOWLEDGEMENT}

This work was supported in part by Xilinx Inc., and by NSF under grants EIA-0321266, CCF-0541363, CNS-0551692, and CNS-0619767.

\section{REFERENCES}

[1] G. Foschini, "Layered space-time architecture for wireless communication in a fading environment when using multiple antennas," Bell Labs. Tech. Journal, vol. 2, 1996.

[2] U. Fincke and M. Pohst, "Improved methods for calculating vectors of short length in a lattice, including a complexity analysis," Math. Computat., vol. 44, no. 170, pp. 463-471, Apr. 1985.

[3] E. Viterbo and J. Boutros, "A universal lattice decoder for fading channels," IEEE Trans. Inf. Theory, vol. 45, no. 5, pp. 1639-1642, Jul. 1999.

[4] B. Hochwald and S. ten Brink, "Achieving near-capacity on a multipleantenna channel," IEEE Trans. on Comm., vol. 51, pp. 389-399, Mar. 2003.

[5] A. Burg, M. Borgmann, M. Wenk, M. Zellweger, W. Fichtner and H. Bolcskei, "VLSI implementation of MIMO detection using the sphere decoding algorithm," IEEE JSSC, vol. 40, no. 7, pp. 1566-1577, Jul. 2005.

[6] L. G. Barbero and J. S. Thompson, "Performance analysis of a fixedcomplexity sphere decoder in high-dimensional MIMO systems," IEEE Conference on Acoustics, Speech and Signal Processing, vol. 4, May 2006.

[7] K. Amiri and J. R. Cavallaro, "FPGA implementation of dynamic threshold sphere detection for MIMO systems," 40th Asilomar Conf on Signals, Systems and Computers, Nov 2006.
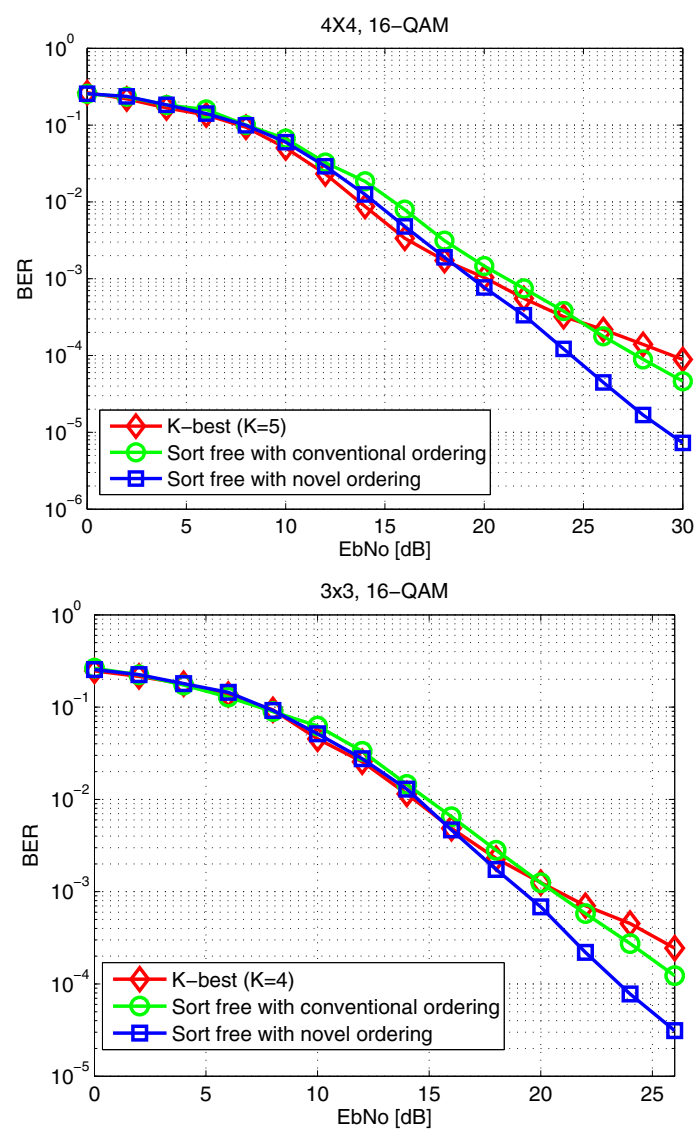

Fig. 4. BER performance of the proposed detector with and without the novel ordering (M-RVD) described in section III-B assuming a 16-QAM modulation for both $M_{T}=M_{R}=4$ and $M_{T}=M_{R}=3$. The $K$-best implementation for $K=5$ and $K=4$ has similar computational complexity as that of the sort-free schemes for $M_{T}=4$ and $M_{T}=3$, respectively.

[8] Z. Guo and P. Nilsson, "Algorithm and implementation of the K-Best sphere decoding for MIMO detection," IEEE JSAC, vol. 24, no. 3, pp 491-503, Mar. 2006.

[9] K. Wong, C. Tsui, R. S. Cheng and W. Mow, "A VLSI architecture of a K-best lattice decoding algorithm for MIMO channels," IEEE Int. Symp. Circuits Syst., vol. 3, pp. 273-276, May 2002.

[10] Z Guo and P. Nilsson, "A $53.3 \mathrm{Mb} / \mathrm{s} 4 \times 4$ 16-QAM MIMO decoder in $0.35 \mu \mathrm{m}$ CMOS," IEEE Int. Symp. Circuits Syst., vol. 5, pp. 4947-4950, May 2005.

[11] M. O. Damen, H. E. Gamal and G. Caire, "On maximum likelihood detection and the search for the closest lattice point," IEEE Trans. on Inf. Theory, vol. 49, no. 10, pp. 2389-2402, Oct. 2003.

[12] A. Burg, "VLSI circuits for MIMO communication systems," $P h D$ Thesis, 2006. 